University of Rhode Island

DigitalCommons@URI

Cancer Prevention Research Center Faculty

Publications

Cancer Prevention Research Center

2010

\title{
A Framework for Incorporating Dyads in Models of HIV-Prevention
}

Benjamin R. Karney

Hyman Hops

Colleen A. Redding

University of Rhode Island, credding@uri.edu

Harry T. Reiss

Alexander J. Rothman

See next page for additional authors

Follow this and additional works at: https://digitalcommons.uri.edu/cprc_facpubs

This is a pre-publication author manuscript of the final, published article.

Terms of Use

All rights reserved under copyright.

\section{Citation/Publisher Attribution}

Karney, B.R., Hops, H., Redding, C.A. et al. AIDS Behav (2010) 14(Suppl 2): 189. https://doi.org/10.1007/ s10461-010-9802-0

Available at: https://doi.org/10.1007/s10461-010-9802-0

This Article is brought to you for free and open access by the Cancer Prevention Research Center at DigitalCommons@URI. It has been accepted for inclusion in Cancer Prevention Research Center Faculty Publications by an authorized administrator of DigitalCommons@URI. For more information, please contact digitalcommons-group@uri.edu. 


\section{Authors}

Benjamin R. Karney, Hyman Hops, Colleen A. Redding, Harry T. Reiss, Alexander J. Rothman, and Jeffry A. Simpson

This article is available at DigitalCommons@URI: https://digitalcommons.uri.edu/cprc_facpubs/33 
Published in final edited form as:

AIDS Behav. 2010 December ; 14(0 2): 189-203. doi:10.1007/s10461-010-9802-0.

\title{
A Framework for Incorporating Dyads in Models of HIV- Prevention
}

\author{
Benjamin R. Karney, \\ Department of Psychology, University of California, Los Angeles, P.O. Box 951563, Los Angeles, \\ CA 90095-1563, USA
}

\section{Hyman Hops,}

Oregon Research Institute, Eugene, OR, USA

\section{Colleen A. Redding,}

Cancer Prevention Research Center, University of Rhode Island, Kingston, RI, USA

\author{
Harry T. Reis, \\ Department of Psychology, University of Rochester, Rochester, NY, USA
}

\author{
Alexander J. Rothman, and \\ Department of Psychology, University of Minnesota, Minneapolis, MN, USA \\ Jeffry A. Simpson \\ Department of Psychology, University of Minnesota, Minneapolis, MN, USA \\ Benjamin R. Karney: karney@psych.ucla.edu
}

\begin{abstract}
Although HIV is contracted by individuals, it is typically transmitted in dyads. Most efforts to promote safer sex practices, however, focus exclusively on individuals. The goal of this paper is to provide a theoretical framework that specifies how models of dyadic processes and relationships can inform models of HIV-prevention. At the center of the framework is the proposition that safer sex between two people requires a dyadic capacity for successful coordination. According to this framework, relational, individual, and structural variables that affect the enactment of safer sex do so through their direct and indirect effects on that dyadic capacity. This dyadic perspective does not require an ongoing relationship between two individuals; rather, it offers a way of distinguishing between dyads along a continuum from anonymous strangers (with minimal coordination of behavior) to long-term partners (with much greater coordination). Acknowledging the dyadic context of HIV-prevention offers new targets for interventions and suggests new approaches to tailoring interventions to specific populations.
\end{abstract}

\section{Keywords}

HIV-prevention; Dyads; Interpersonal relationships; Safer sex; Models

(c) Springer Science+Business Media, LLC 2010

Correspondence to: Benjamin R. Karney, karney@psych.ucla.edu. 
HIV is contracted by individuals, but in the majority of cases it is transmitted in dyads [1]. In the United States, $73 \%$ of HIV transmission occurs through sexual contact within gay and heterosexual couples [2]. Worldwide, the primary route of transmission is heterosexual sex [3]. Preventing the spread of HIV therefore requires targeting behaviors that are inherently dyadic and relational (i.e., behaviors that are coordinated and enacted with another person).

Given the centrality of dyadic processes to HIV transmission, the goal of this paper is to propose a framework that incorporates dyads and relationships within models of HIVprevention. Toward this end, this paper is organized in five sections. The first section briefly summarizes current prevention efforts that address dyadic processes directly. To guide the design of new intervention strategies that build on these efforts, the second section presents a framework that specifies how models of dyadic processes can inform models of HIVprevention. The third and fourth sections highlight links between the dyadic processes that are the focus of our framework and both individual-level and structural-level processes as they relate to HIV-prevention. The final section draws out the implications of our framework for future research and intervention strategies.

A premise of the analysis that follows is that, because transmission of HIV most frequently involves two people (i.e., one to transmit and one to receive), the $d y a d$ represents a crucial unit of analysis for prevention efforts. Kelley et al. [4]. characterize dyads in terms of the extent to which each partner influences the other's thoughts, feelings, and actions. In other words, Person A has some degree of influence on Person B, who in turn has some degree of influence on Person A, and so on in a reciprocal sequence. By focusing on mutual influence, a dyadic perspective does not suggest that people's behavior is unaffected by their own attributes, nor does it require that partners always influence each other. Rather, this perspective posits that behavior can be jointly determined by personal attributes as well as the influence of another person [5].

It follows from this definition that a dyadic perspective on HIV-prevention is not the same as a relationship perspective. All relationships are dyadic, but not all dyads are relationships. Relationships have a temporal perspective, involving the partners' history of interaction with one another and an imagined future [6]. In relationships, partners influence each other mutually, frequently, and in diverse ways [7]. Within a dyadic perspective, then, there can be a continuum of involvement and influence ranging from superficial (one-time encounters) to enduring (long-term relationships). The framework we outline here is designed to address dyads all along this continuum.

A range of behaviors are included in the category of safer sex, such as choosing to have sex with multiple partners or a single partner, getting tested for HIV and communicating the results, and using condoms correctly. All of these behaviors are affected by dyadic processes, but the emphasis here is on predicting those behaviors that most directly involve the coordination of two people (e.g., using condoms), where coordination in this context is defined as the process through which two individuals act together to achieve a specific goal. 


\section{Dyads in Existing HIV-Prevention Efforts}

Most efforts to promote safer sex in the United States focus exclusively on individuals. Although meta-analyses indicate that these interventions are generally effective [8-13], there is consensus that sexual health interventions are not nearly as effective as they could be. In particular, these interventions have had markedly less effect on the behavior of individuals involved in ongoing, intimate relationships [14]. For example, among men who have sex with men (MSM), the group at highest risk for contracting HIV in the United States, $68 \%$ of HIV infections are transmitted from primary sex partners [15]. Recognizing the dyadic context of HIV transmission has led to calls for broadening HIV-prevention efforts to address not only both partners in sexual dyads, but the relationship between the partners as well [16-19].

Interventions can account for the dyadic context of HIV-prevention behaviors in at least four ways. First, programs that target individuals can refer to and acknowledge dyadic factors. For example, an intervention may recognize that different strategies for introducing condoms may be more or less appropriate depending on the kind of relationship(s) individuals have with their sexual partners. This is the default approach for intervening with individuals who are not in committed relationships, or when both members of a couple are not available. Second, interventions can attempt to provide both members of couples with the same materials. The goal of this approach is efficiency (i.e., it is easier to ensure that both partners have the right information if it is presented to both of them directly). Third, programs can explicitly train couples in specific strategies and skills for coordinating safer sex that are responsive to features of the relationship. The goal of this approach is not only efficiency, but increased effectiveness. For example, if couples are encouraged to practice talking about safer sex, HIV testing, and condom use, presumably they will be better at doing so when it matters most [20]. Fourth, programs can directly promote improved relationships independent of safer sex behavior. The rationale for this approach is that stronger, closer relationships between sexual partners may provide a context that supports more effective coordination of safer sex as well as safer sexual practices.

Existing couple-focused interventions in the U.S. usually adopt a mixture of these approaches, focusing primarily on the attitudes and behaviors of individuals, but also addressing patterns of communication in couples to varying degrees. For example, interventions with serodiscordant heterosexual couples[21-25] generally build upon HIV counseling and testing protocols designed for individuals but present comparable information to both partners in a couple simultaneously (the second approach).

Interventions that directly train couples to discuss issues related to HIV-transmission and prevention (the third approach) are less common, but several recent efforts have been moving in this direction [14, 18, 26-28]. For example, Project CONNECT [14], one of the first studies to examine couples-focused STI/HIV prevention, found that an intervention that directly trained women, and in one group their partners as well, to communicate about condom use (the first and third approach) led to more consistent condom use, compared to a control condition that did not include such training. Another study entitled PARTNERS (Partners Against Risk-Taking: A Networking, Evaluation, and Research Study), designed to 
help heterosexual partners avoid unwanted pregnancy and sexually transmitted infections (STIs), also found that direct training in discussing safer sex with a partner was associated with increases in condom use efficacy relative to an education-only control group [27, 28].

Although HIV-prevention interventions have begun to include both members of couples [14, $27,28]$, to date these interventions have seldom addressed the relationship between the partners directly (the fourth approach). One recently developed intervention that does address relationships directly is Project Eban, the NIMH Multisite HIV/STD Prevention Trial for African American Couples [21]. This project is evaluating a culturally congruent couples-based intervention [22,23] that includes theory-based components addressing communication, sharing, sexual risk and eroticizing condom use, planning for the future, and relapse prevention. The Eban intervention incorporates not only comparable information, communication, and skills building exercises for both partners, but also components that focus on relationship enhancement.

Yet even the designers of couples-focused HIV-prevention interventions recognize that incorporating relationships into HIV-prevention programs raises more questions than existing studies have answered. As Kraft and colleagues [28] conclude, "Future research should also identify conditions under which couples interventions might be effective by exploring the optimum mix of individual psychosocial and relationship factors to address for different types of couples, whether to explicitly address relationship factors, and whether to target individuals or couples."

A significant obstacle to addressing these questions and refining dyadic HIV-prevention strategies is the lack of models to guide research and practice. Many HIV-prevention models focus on behavior and behavior change at the individual level [29-31]. Although the application of these models recognizes variability in the types of relationships people are in, dyadic processes are not specified in these models. Yet sophisticated models of dyadic influence and interpersonal processes exist and have been put to good use in explaining important and complex outcomes in other domains, such as conflict resolution, divorce, and mental and physical health of spouses and partners [32]. A natural direction for theory development in HIV-prevention, therefore, is to integrate these two existing theoretical perspectives.

\section{A Dyadic Framework for HIV-Prevention}

To expand the theoretical foundation of HIV-prevention efforts, we propose a framework that integrates models of HIV-prevention with existing models of dyadic processes. One observation motivating this framework is that sexual behavior requires varying degrees of coordination between two people, where the extent of coordination depends on the relationship between the partners. In anonymous dyads (e.g., virtual strangers), there is limited mutual influence between partners, which limits the opportunity to coordinate activities that might result in safer sexual practices. In long-term intimate relationships, partners often have substantial mutual influence, so better coordination is both possible and necessary. The framework described here suggests that a clear understanding of the dyadic 
context of the behavior should determine which variables are most relevant for predicting the likelihood that a couple sharing a sexual encounter will successfully engage in safer sex.

\section{The Centrality of the Dyadic Interaction}

To account for the way two individuals succeed or fail to coordinate safer sex, the framework we propose describes sources of influence on safer sex that range from extremely proximal to the dyad (e.g., the partners' immediate physical environment) to extremely distal (e.g., the cultural and historical context in which the behavior is occurring). There are many precedents for this organization, one notable example being the concentric rings of Bronfenbrenner's [33] social ecological models of child development. Following in this tradition and building on the premise that safer sex behavior is the direct result of a dyadic process, the framework depicted in Fig. 1 arranges the sources of influence on that process along a continuum ranging outward from the dyad.

At the center of the framework is the core proposition that safer sex between two people is regulated by a dyadic capacity for successful coordination. When sex is imminent and transmission of HIV becomes a possibility, some couples will be able to coordinate their actions effectively to achieve a shared goal of mutual protection, whereas some couples will not coordinate effectively and others will be unable to coordinate safer sex at all (e.g., anonymous sexual encounters). Coordination in this sense need not be explicit or verbal. Even when one partner silently opens and puts on a condom, the other partner has been made aware of the first partner's intention and tacitly consents.

It follows from this proposition that anything that affects the enactment of safer sex ultimately affects or is affected by the dyadic interaction (i.e., the mutual exchange of behaviors between two people) through which sexual behaviors are coordinated. Kelley et al. [7] make a similar point in their comprehensive analysis of dyadic outcomes, arguing that interaction is the common pathway through which qualities of each partner, the extent of their mutual influence, the environment, and the broader culture all affect the dyad. Dyadic interaction, therefore, must be considered in any model of safer sex outcomes.

The remainder of the framework organizes the proximal and distal sources of influence on the dyadic interaction. In addition to the nature of the relationship between the individuals, the framework recognizes two proximal sources of influence that have been featured in prior models of HIV-prevention. One is the beliefs and motivations of each partner. All else being equal, partners who believe in and care about preventing HIV transmission are more likely to try to enact safer sex behaviors than those that do not. The second is the immediate physical environment in which the interaction takes place. Coordinating the use of a condom, for example, requires that condoms are available. Thus, features of the environment may moderate how or whether the dyadic interaction results in safer sexual behavior. Given that both of these sources of influence have been described in detail elsewhere [34], we will not elaborate here. Instead, our emphasis is on aspects of the dyad that have been overlooked in existing models, specifically the ways in which the capacity of the couple to coordinate safer sex practices effectively is facilitated or constrained by the nature of the relationship between the partners. The framework described in Fig. 1 suggests explicit predictions about when that relationship will play a larger or smaller role in 
accounting for safer sex behaviors. Specifically, the greater the ongoing mutual influence between the partners, the more their relationship will shape their capacity for coordinating safer sex. The less the ongoing mutual influence, the more their capacity for coordinating safer sex will depend on the individual motivation and ability of each partner and their immediate physical environment.

Moving outward from the immediate context of a sexual encounter, stable characteristics of individuals can affect the dyadic capacity to coordinate safer sex in two primary ways. First, qualities of the individual naturally affect personal motivation and the ability to engage in safer sex practices. For example, individuals who are more conscientious and engaged with the issue of HIV infection may be more likely to recognize when they are at risk for contracting and transmitting HIV and, thus, should be more motivated to practice safer sex than those who are not. Second, our framework suggests that individual characteristics may also affect the capacity to coordinate safer sex through their effects on the relationship and social interactions between the partners. For instance, individuals who fear conflict with their partners (due to low self-esteem, dependence on the relationship, or a history of abuse) may be unable to coordinate safer sex, regardless of their motivation to do so. In contrast, those who are knowledgeable, assertive, and self-confident may be able to influence their partners to engage in safer sex, even if their partners are initially unwilling to do so. Individual differences in adult attachment [35] should also impact the dyad's capacity to coordinate behaviors. For example, anxious attachment, which is manifested as inappropriate worry about a partner's availability and regard, may lead people to defer to their partner's wishes, either to gain acceptance or avoid rejection. This may result in either safe or unsafe sexual practices, depending on the partner's preferences. Avoidant attachment, which is associated with emotional flattening and discomfort with intimacy and closeness, typically leads to less commitment, emotional "game-playing" in relationships, and less concern about the partner's (or one's own) well-being, all of which may also increase unsafe sexual behavior.

At the most distal level, the framework describes structural-level variables that moderate the ways that each of the myriad sources of influence on the dyadic interaction affect each other. Culture, for example, constrains the way partners may communicate about sex. In cultures that allow sexual openness, the relationship between the partners ought to play a larger role in how interactions unfold, whereas in cultures that prohibit open communication about sex, dyad members should have less flexibility to shape their interactions. The social structure, in particular the relative power of men and women [36,37], should also constrain how partners are able to interact.

\section{Interpersonal Influences on Dyadic Interaction}

Because relationships have not been featured in prior HIV-prevention models, the ways that relational contexts can affect dyadic processes deserve elaboration. Past research has examined several aspects of relationships that might affect the ability of one or both partners to coordinate their behaviors to achieve shared goals. To provide a flavor for this research, we briefly describe six elements of relationships that may be particularly relevant to coordinating safer sex. 
Trust-Trust entails the expectation that a partner will support one's best interests, even when they conflict with the partner's own personal interests. It is the foundation upon which meaningful relationships are built [38]. Higher levels of trust are likely to foster more open, direct, and honest communication (e.g., about HIV-relevant risks and behaviors), and may also motivate individuals to put their partner's welfare and well-being ahead of selfinterests. Trust also makes partners more willing to be influenced by the other given that the other is presumed to have altruistic intentions. In relationships, trust tends to be inferred from situations in which the partner has had to choose between self-interest and sacrificing for the good of the other. Because this type of history is much less likely to characterize short-term interactions or newly formed relationships, partners in this case must decide whether or not to trust the other on the basis of personal predispositions and situational factors. The decision to trust a partner may also interfere with safer sex behaviors for individuals who see no need to engage in safer sexual practices with "trustworthy" partners.

Intimacy-Interactions are intimate to the extent that partners are open and honest about themselves and responsively supportive to the other. Intimate interactions generally feel personal, authentic, and meaningful, and lead partners to feel understood, valued, and cared for [39]. Empathy and empathic accuracy are also related to the development and experience of intimacy [40]. When partners feel intimately responded to, they tend to be more open about their feelings and preferences and they are more responsive to their partner's feelings and preferences, even in threatening, awkward, or difficult interactions (e.g., whether or not to use a condom, asking about sexual history and practices). This pattern of interaction should facilitate partners' ability to coordinate their behaviors toward shared goals. Lack of intimacy, in contrast, may cause partners to be unwilling or afraid to discuss these situations, which may lead to inaccurate assumptions about the other's HIV status or safer-sex practices.

Satisfaction-Relationship satisfaction has been defined in two ways: as positive affective experience, or as need fulfillment [41]. Both kinds of relationship satisfaction are directly relevant to safer sexual practices. Positive affect is intrinsically reinforcing, builds social resources, and engenders caring and concern for others. If personal need fulfillment is high, partners should be more willing to try to fulfill each other's needs. Perhaps more importantly, people in satisfying relationships tend to be concerned about protecting their partners' well-being, such as by practicing safer sex. Many researchers also view relationship satisfaction as central to good communication. All other things being equal, in satisfying relationships, people communicate more openly about sex and should be more responsive to their partner's influence attempts regarding safer sex practices.

Commitment-Commitment refers to the intention to continue in a relationship [42]. In more committed relationships, each partner has a stake in the other's well-being; in less committed relationships, individual goals take priority. As a result, committed partners are motivated to be more accommodating, more willing to sacrifice, more concerned about their partner's wishes, and they are more likely to think of their partner's goals and outcomes to some extent as their own [42, 43]. For this reason, commitment typically fosters relationship-maintaining behaviors, which in turn cultivates continued commitment. Persons 
in committed relationships should care more about protecting their partner's HIV-status and finding ways to keep their partner's sexual needs satisfied with safer sex practices. The level of commitment between partners should moderate the effectiveness of different strategies of intervention: in less committed dyads, individual-centered approaches to prevention may be more effective, whereas in committed dyads, relationship-centered approaches may be warranted.

Communication-Communication, which occurs through both verbal and nonverbal channels, is essential to all interactions. All of the relationship factors already described are thought to operate in part through their effect on how partners communicate with each other [7]. Several features of communication process are particularly relevant to sexual interaction: interdependence, or the degree to which partners influence each other; symbolism, or the meaning that each communicative act conveys to the other; and ambiguity, or the degree to which a communication has clear interpretation. Of particular importance are the conditions that allow partners to more or less accurately infer what their partners are thinking and feeling. This includes empathic accuracy and deception, both of which should be highly relevant during sexual (and potentially sexual) interactions. How these processes unfold determines whether participants in a sexual encounter are likely to be informed and able to enact appropriate safer sex practices.

Power-Power, which is the ability to influence another person's behavior without being influenced in return, varies from one dyad to another [44]. Some dyads are egalitarian in the sense that each partner expects to be heard and to have a "say" in all joint activity. Other dyads are more asymmetric (non-egalitarian), with one partner having more influence than the other. Several factors predict power asymmetries, including personal resources, social roles, personality traits, and culturally based gender roles. Typically, the higher power partner in a relationship has greater influence over decisions about the type of sex that is engaged in or whether safe sex practices are followed. Higher power partners, therefore, may need to be the primary "targets" of couple-based HIV interventions. The lower power partner, however, may be more motivated to conceal relationship-threatening information relevant to HIV-transmission, so that different interventions might be directed at lower power partners.

This list is not intended to be exhaustive. Many other elements of interpersonal relationships are known to influence dyadic processes in couples [45]. Nor are these processes independent of each other. For example, greater trust promotes intimacy, which in turn may enhance relationship satisfaction and ultimately greater commitment, which may then feed back on trust [38]. The main point of this brief review is simply to highlight that each of these elements could affect HIV-prevention by influencing the effectiveness of a couple's ability to coordinate or negotiate safer sex behaviors. For this reason, consideration of the specific qualities of the relationship between the partners within a sexual dyad is crucial for understanding and altering the likelihood of safer sex practices. 


\section{Applying the Model: The Example of Concurrency}

To illustrate how the current framework might organize and elaborate upon existing efforts at HIV-prevention, we can apply the framework to a specific phenomenon: concurrency, or engaging in sexual activities with multiple partners. Internationally, rates of HIV transmission are markedly higher in countries and cultures where concurrent sex partners are more accepted within primary relationships. For example, within the U.S. MSM couples are more likely to accept and agree to have concurrent sex partners [46]. Indeed, one study of mixed-serostatus MSM couples reported that $71 \%$ had extradyadic sex [47]. Among US adult heterosexuals, concurrency has been an important source of some localized epidemics $[48,49]$.

The current framework suggests several different ways of addressing concurrency within HIV-prevention interventions. To begin, from the perspective of a specific dyad in a position to coordinate safer sex behaviors, whether or not each partner is having sex with other partners is part of the relational context of the couple. One use of the framework is to highlight other elements of the relational context that make concurrency more or less likely. For example, couples who are in a committed, satisfied relationship may be less likely to engage in extradyadic sex than couples who are less committed and unsatisfied with the relationship. Interventions that promote or improve these aspects of relationships may reduce this source of risk for HIV transmission indirectly.

The framework also highlights relational features that may account for whether or not members of a dyad are aware of each other's concurrent partners. One recent study of concurrency among 90 adolescent African-American heterosexual couples found that 16$37 \%$ of individuals who perceived that their partners were sexually exclusive were involved with partners who were not exclusive when interviewed separately [50]. In this study, elements of the relationship predicted accuracy. Partners in relationships longer than 6 months and partners reporting more closeness were more likely to report each other's concurrent activities accurately [50]. Thus, intervention strategies that increase trust and improve communication may put partners in a better position to acknowledge each other's concurrent partners and take appropriate precautions, even if they do not target concurrency explicitly.

The framework also acknowledges how culture is likely to shape the relational context that surrounds specific dyadic interactions. Cultural values strongly influence preferences and practices regarding concurrent sex partners, both within the United States and in other countries. For example, in countries with Islamic influences, men typically have multiple wives (co-wives) [51, 52]. In cultures where monogamy and sexual exclusivity are normative and encouraged, improving the quality of the relational context may reduce concurrent sexual partnerships, thereby reducing the risk of HIV-transmission. In cultures where concurrency is accepted or tolerated, however, the relevant elements of the relational context may be those tied to the ability of the dyad to take appropriate precautions, such as power differentials or the extent to which each dyad member is invested in the other's health and well-being. 
Although the framework presented in this paper does not address all of the challenges posed by concurrency, it provides a starting point for specifying how factors that operate at different levels of analysis (i.e., cultural, individual, and relational) may fit together to help explain this behavior. By identifying different paths of influence linked to concurrency, the framework suggests specific directions for interventions and ways of tailoring different interventions to different target groups.

\section{Integrating Dyadic and Individual Perspectives on HIV-Prevention}

In contrast to the dyadic framework outlined here, virtually all current models of HIVprevention focus on individual-level variables that specify how characteristics or attributes within one person (e.g., her or his thoughts, feelings, and perceptions) predict that person's behavior [29-31]. The Theory of Reasoned Action [53] provides an illustrative example. According to the TRA, an individual's volitional behavior is a function of her or his intention to engage in the behavior, which in turn is a function of her or his attitude toward the behavior along with the perceived social norms for performing it (both are aspects of an individual's motivation and beliefs, represented in Fig. 1). Behavior, in other words, reflects a process of deliberation (as people form an intention based on their attitude and extant social norms) followed by a process of implementation (as people translate their intention into a behavior). Although social norms capture an individual's perceptions of what other people believe one should do in a given situation, all of the pathways specified in the TRA capture relations between constructs within a single person; the potential influence of the attitudes, norms, or intentions of anyone else (e.g., a relationship partner) is not represented.

Yet the processes specified in individual-based models can easily be embedded within more general dyadic models, such as the one described by Fig. 2. Because people usually make decisions and take actions in the company of others, their behavior often requires coordination with another person. Imagine, for example, two people who have just started dating and are deciding whether or not to be tested for HIV. An individual-level framework such as the TRA assumes that each individual's behavior is a function of only her/his own personal beliefs and perceptions. In Fig. 2, this hypothesis is depicted by solid lines. To the extent that the two individuals, still in a new relationship, have not yet begun to influence each other's decision-making with regard to HIV testing, their personal beliefs should have little if any influence on the other's behavior. In this case, the processes specified by an individual-level model should be sufficient to explain how each partner's attitudes and subjective norms are related to his or her own intentions and, in turn, HIV-relevant behavioral outcomes.

Individual-level models, however, do not fully capture influence processes when relationship partners influence each other, either unilaterally or mutually. As shown in Fig. 2 , a dyadic model of behavioral intention allows for "reciprocal influence" in which each partner's attitudes, subjective norms, and intentions can potentially affect his/her partner's intentions and behaviors, above and beyond how each partner's personal attitudes and subjective norms affect his/her own personal intentions and behavior. With respect to behavior, dyadic models acknowledge that the coordinated actions of a couple will be the result of some combination of the behavioral intentions of both partners. Partner effects 
index the extent to which an individual's outcomes (e.g., behavior) are predicted by the attributes (e.g., attitudes, subjective norms) of his/her partner, statistically controlling for the individual's own scores on the same attributes. As such, they represent the statistically independent effect that relationship partners have on one another. Acknowledging these effects is a novel feature of dyadic models such as the Actor-Partner Interdependence Model (APIM) [54].

According to a reciprocal dyadic model, an individual who has negative attitudes toward HIV testing, does not perceive strong normative pressures to get tested, and thus has no intention of getting tested may still be more likely to get tested if his/her partner has-and clearly expresses-positive attitudes about testing, harbors strong perceived norms for doing so, and thus intends to get tested. In this case, an individual-level model would have difficulty explaining why this individual would not simply act in line with his or her own negative personal attitudes, norms, and intentions. The individual may get tested primarily in response to his or her partner's communicated attitudes, wishes, and intentions. A dyadic perspective also expands on individual-level models of behavior through the novel premise that people within a relationship may choose to act based on how they think and feel about their partner and relationship, independent of their direct thoughts and feelings about the behavior. For example, a person may choose to engage in safer sexual behavior because s/he loves or feels committed to his/her partner, not necessarily because s/he likes or feels favorable toward using condoms. It is important to emphasize that dyadic models do not require the existence of partner or relationship effects. They merely provide a statistical framework for measuring and modeling these effects if they exist.

In addition to acknowledging that individual-level constructs may exert cross-partner effects, a dyadic perspective also highlights new constructs with the potential to expand our understanding of the factors that regulate how and why partners make certain HIV-relevant decisions. For example, according to traditional individual-level models, subjective norms reflect what an individual perceives as "modal" standards, expectations, and/or practices regarding how one ought to behave in a particular situation. These general perceived norms, however, may differ from those that develop within an established relationship. For example, even though an individual may live in a culture or environment in which HIV testing is frowned upon, s/he and his/her partner may agree that regular testing is good for their relationship. Consistent with the principle of correspondence [55], if a relationshipspecific norm regarding HIV testing develops, it may then govern the behavior of each partner more strongly than the generalized perceived subjective norms that each partner harbors. Measuring subjective norms at different levels may be especially important in predicting and understanding how partners make decisions about HIV-relevant behaviors in different relational contexts.

A dyadic perspective can also distinguish between the effects of partner beliefs and the effects of an individual's perceptions of what his or her partner believes, as depicted in Fig. 3. The accuracy with which partners know one another's intentions is likely to depend on certain aspects of their relationship, such as the degree of trust, commitment, satisfaction, etc. Over time, partners in long-term relationships may learn to anticipate or forecast the intentions of their partners better, and these "internalized" partner intention perceptions may 
then guide how an individual acts in specific situations. Indeed, sometimes perceptions of what a partner wants may guide an individual's decision-making and behavior even more strongly than what the partner truly desires.

By adopting a dyadic approach, investigators may also become more aware of certain important features within relationships that could affect how they derive HIV-relevant hypotheses. For example, most individual-level models of behavioral decision-making have focused on an individual's own beliefs about the behavior in question (e.g., whether or not to use condoms), which have motivated the development of intervention strategies designed to: (a) promote more favorable attitudes toward the behavior, (b) heighten people's confidence in their ability to execute the behavior, and (c) render the behavior more socially or normatively appropriate. A dyadic perspective does not dismiss the importance of these classes of beliefs; rather, it introduces the novel premise that people may choose to act within a relationship based on how they think and feel about their partner and relationship. Figure 3 illustrates how unique beliefs and feelings about the relationship harbored by each partner could affect their behavioral intentions. For example, Partner A's beliefs or affect about the relationship may guide his or her behavioral intentions more strongly than do his/her personal attitudes or social norms enroute to predicting what he or she does with respect to a given HIV-relevant behavior.

Dyadic models also allow for the measurement and modeling of dyadic variables, such as power, intimacy, trust, and communication patterns, which are likely to moderate the extent to which partners affect and are affected by each other. One particularly important moderating variable is likely to be relative power within the relationship. When one relationship partner has greater power than the other, the more powerful partner can exert greater influence on dyadic decisions [56]. In this situation, the more powerful partner in the dyad exerts greater influence on the behavioral outcomes of both partners. In statistical terms, within-dyad power differentials should be reflected in significant actor/partner statistical interactions.

A dyadic model also allows one to capture a wider range of factors that could affect the patterns of communication between two individuals. For example, the style and frequency of communication between two people is likely to moderate the extent to which partner effects are observed. If both partners openly share their personal thoughts and feelings about safe sex practices, partner effects should be stronger. Over time, more open communication may make the partners' beliefs and behavior more similar. If there are power differences between the partners, however, patterns of communication that allow the more powerful partner to dominate the discussion should enhance the higher power partner's impact on what the lower power partner does. In a similar manner, differences between partners in personality and socio-demographic variables that affect the style or frequency with which people interact may also moderate predicted relations between constructs that can be specified and tested within a dyadic model.

Dyadic models are equally applicable to HIV-prevention behaviors that each partner engages in separately (e.g., deciding whether or not to get HIV testing) or that partners make jointly (e.g., deciding whether or not to use a condom). In both situations, the behavioral 
outcomes still depend on beliefs held by both dyad members. And to the extent that there are power differences between the partners, the intentions of the more powerful partner should have a stronger impact on either behavioral outcome.

\section{Integrating Dyadic and Structural Perspectives on HIV-Prevention}

In acknowledging the dyadic context of HIV transmission, our analysis focuses on the nature of the relationship between two individuals. However, dyads are also affected by the broader social structure within which they are embedded. As described in Fig. 1, structural factors can have a direct effect on safer sex behaviors through, for example, cultural influences on each partner's knowledge and motivation and environmental influences that facilitate or constrain safer sex practices. Our interest here, however, is on how structural factors may alter the nature of the dyadic interaction around safer sex. Although there are many structural factors that may influence HIV risk behaviors, such as high-risk neighborhoods, poverty, drug use availability, etc., here we focus on three elements with specific implications for dyadic processes: the family, the peer group, and race/ethnicity. In this section, we examine how each of these structural sources of influence may shape the dyadic capacity for successful coordination of safer sex behaviors and, hence, could be addressed directly within HIV-prevention efforts.

\section{Parents and Other Family Members}

Half of all persons with HIV became infected during adolescence and young adulthood [57] when family influences may play a significant role. Although family influences are noteworthy during childhood and adolescence, there is increasing evidence that parental influence continues into young adulthood and beyond even after the offspring have left the parental home [58-60]. And with the increasing availability of cell phones and internetbased modes of communication the frequency of interaction between members of extended families continues to grow [61]. Thus, identifying familial variables that influence high-risk sexual behavior may provide clues about the intervention/prevention activities that might reduce the prevalence of infection not only in adolescence but throughout young adulthood.

The influence of parents and family members on sexual behavior is substantial, but it also tends to be indirect, given that parents and family members are typically absent when sexual interactions occur. The framework described in Fig. 1 suggests that parents and family members can affect the enactment of safer sex behaviors through their direct influence on the way individuals choose and interact with their sexual partners. Indeed several studies have documented an association between positive parent-adolescent interactions and interactions between young people and their peers [62]. When parent-adolescent communication has been strong and effective, adolescents should be able to communicate more directly and effectively with their peers and potential partners, allowing them to be more effective at coordinating safer sexual practices. Fasula and Miller [63], for example, found that good mother-daughter communication in African-American families delayed the onset of sexual intercourse in high-risk environments (i.e., for those who perceived that their friends were sexually active). Similar effects have been found in studies of Hispanic families [64]. Perrino et al. [65] suggest that improved parent-adolescent communication provides a context that not only promotes positive changes within the family, but may also generalize 
to other settings resulting in healthier relationships with peers, greater intimacy, and more effective communication about sexual issues outside the family. Thus, HIV-prevention efforts that directly target parent-child interactions may have long-term indirect benefits for the ability of sexual dyads to communicate effectively about safer sex.

\section{Peer Groups}

Early in the development of individual's sexual activity, peer groups assume a significant role in shaping a dyad's capacity to coordinate safer sex behaviors. This structural element affects that capacity through several pathways, a number of which have been explored extensively by researchers focusing on HIV-prevention. For example, consistent with the TRA, peer groups are thought to be important sources of an individual's perceived norms about sexual behavior, such that if individuals believe that their peers behave in sexually risky ways, they perceive tacit approval and perhaps encouragement to behave similarly [66]. In the moment of an impending sexual encounter, those norms are likely to shape whether and how effectively couples communicate about safer sex. Additionally, peers outside the intimate partner relationship can also be influential in shaping the context of sexual behavior. For example, alcohol and drug use among adolescents and young adults is strongly associated with the level of alcohol use in one's peer group [67-70]. Membership in a peer group in which substance abuse is frequent or normative thus sets the stage for sexual interactions to occur in contexts in which both partners' capacities to coordinate safer sex effectively is constrained by intoxication and impaired judgment.

Peer groups also affect the dyadic capacity to coordinate safer sex through direct effects on the nature and quality of dyadic relationships [71]. A number of studies have described substantial continuity between the quality of individuals' relationships with their peers and the quality of their subsequent sexual and intimate relationships [72-75]. Moreover, adolescents and young adults adopt codes of conduct within their own relationships that they have observed in the relationships of their peers [76]. It follows that individuals who can communicate openly about sexual behavior with their peers should have a greater capacity to communicate effectively about safer sex with their sexual partners, and should thus be more likely to enact these behavior during their own sexual encounters.

Considering the influence of peers in this way suggests several directions for involving peer groups within HIV-prevention efforts. Programs have already focused on changing norms within adolescent and young adult peer groups. The current framework suggests that interventions to promote effective communication among peers may also be effective at promoting communication within sexual dyads, even if those dyads are not targeted directly. Such interventions may be especially appropriate in communities that resist direct sexual education for youth.

\section{Race, Ethnicity, and Culture}

HIV infection rates vary widely across ethnic and racial groupings. African-Americans, for example, have the highest proportion of newly diagnosed HIV (51\%), more than all other groups combined and among black females, more than $80 \%$ of transmissions occur through heterosexual contact [77]. Hispanics have the second highest rate of AIDS [78], with 
Hispanic adolescents accounting for $20 \%$ of all people with that diagnosis and a disproportionate number of cases among Latinas. It has already been recognized that intervention or prevention efforts must be tailored to the specific needs of each of these groups. The current framework further suggests that acknowledging the effects of cultural differences on the dyadic interaction is likely to be crucial for the success of interventions aimed at any specific ethnic or racial group.

In particular, cultural norms and scripts relating to gender roles and power are likely to play a role in constraining or enhancing discussions of safe sex practices among some ethnic or racial groups [79]. Among Latinos, for example, where early sexual initiation, multiple partners, and less condom use are all more prevalent than in non-Hispanic Whites [80-82], strong cultural norms of machismo and marianismo act against efforts to promote frank discussions of sexual behavior in Hispanic families [83, 84] and couples [85]. The development of HIV-prevention efforts that integrate cultural variables in their designs [66, 84, 86, 87] reflects a recognition of the powerful roles that racial and ethnic group membership play in shaping sexual behavior. Yet studies exploring how race/ethnicity may moderate the effects of relational and social variables on risky sexual behavior remain rare [88]. Future research in this vein would benefit from the development and testing of models that can specify the interplay between cultural, dyadic, and individual factors. In particular, the degree to which certain cultural differences may promote or inhibit dyadic processes believed to affect patterns of safer sex behaviors enacted by individuals is a logical and important next step.

\section{Implications of a Dyadic Framework for HIV-Prevention}

In this concluding section, we discuss some of the most significant implications of adopting a dyadic perspective for developing better and more effective HIV-prevention programs and interventions.

\section{Refining and Expanding Interventions}

The framework described here has two major implications for interventions aimed at HIVprevention. First, tailoring interventions to specific populations must take into account the relationship between the two partners in a given dyad. The nature of that relationship is likely to moderate the effectiveness of different strategies for promoting safer sex. For example, to the extent that an individual or a target population frequently engages in anonymous or short-term sexual encounters (i.e., there is a minimal relationship between dyad members), individual-level interventions should be more effective than those that focus on the dyad-level. If, however, an individual or target population typically has sex within established, intimate relationships, the framework suggests that the qualities of that relationship should directly facilitate or constrain the dyadic capacity to coordinate safer sex. Accordingly, interventions that acknowledge the relationship within the dyad should be more effective. Even within populations of couples, however, knowing about the relationship context should be crucial for planning interventions. Those in highly conflictual, distressed, or abusive relationships should be less likely to benefit from interventions that require dyads to practice communicating. In fact, such programs may actually backfire. Future research needs to identify the minimal level of relationship functioning necessary for 
a dyad to benefit from relationship-focused HIV-prevention strategies. And because the same relationship can change across time (i.e., in commitment, satisfaction, trust), the same dyad may benefit from different strategies at different stages of the relationship.

Second, the current framework highlights possible targets of intervention that would not be obvious for interventions guided by purely individual-level or structural-level perspectives. As noted earlier, with few exceptions, even current HIV-prevention strategies that explicitly target dyads seldom address aspects of the dyadic relationship directly. The current framework suggests that interventions could be expanded and refined by explicitly addressing and aiming to enhance features of the relationship that are likely to influence the dyadic capacity to coordinate safer sex behaviors. By emphasizing aspects of the dyad, we do not mean to argue that aspects of the relationship between the partners will be appropriate targets of intervention in all cases. Sometimes it may be enough for one partner to take appropriate action (e.g., by getting tested for HIV, by refraining from extradyadic sex, by abstaining from sex altogether). Yet even individual-level interventions can address relationship issues, given that individuals might be able to apply the lessons learned from these interventions in their future relationships. The inverse is also true: Interventions that focus on dyads also need to address non-relationship variables (e.g., attitudes, self-efficacy, motivations) that traditionally have been the focus of individual-level interventions. The combination of the two approaches may prove synergistic by instilling in individuals the beliefs and motives to practice safer sex, and then strengthening the relational context so that dyads can coordinate their safe sex practices better and more successfully in order to achieve their ultimate goals.

\section{Methodological Issues in HIV Research on Dyads}

Conducting research on basic processes and intervention effectiveness in HIV prevention is complicated enough, but acknowledging the centrality of the dyad raises several unique methodological challenges that the next generation of researchers will have to face. For example, intervention programs aimed at dyads may find that successfully recruiting two individuals is more than twice as difficult as recruiting one individual. Once one partner in a dyad has been recruited, the other partner is of unique importance, especially with respect to studying HIV outcomes. Similarly, although minimizing attrition is a challenge for any research that follows dyads over time, the possibility that dyads will dissolve their relationship and lose motivation to continue participating in a study of "couples" poses additional challenges to retention that research focusing on only individuals avoids.

Research using dyads also places unique demands on measurement and data analysis. With respect to measurement, the ability to collect data from both members of a dyad raises the likelihood that each member may provide different answers to the same questions. For example, relationship partners may have very low agreement about specific behaviors they report having engaged in, even during the past 24 hours [89]. To the extent that such selfreports are important outcome variables for research on HIV prevention (e.g., "Did the two of you use a condom the last time you had sex?"), dyadic data can confront researchers with several important choices when partners provide different answers [90]. The researcher may decide a priori that one partner is the more reliable informant, may randomly choose one 
answer over another, or may create decision rules such as only accept responses when both partners agree.

With respect to data analysis, whereas most statistical methods assume independent data units, data provided by two people in a relationship are not statistically independent. In a study of 100 individuals, each of whom is partnered, there may be data from 200 people, but there are only 100 "independent" units of analysis. Techniques for addressing the dependence of data from two members of a dyad have been developed [54], but researchers who are unfamiliar with dyadic data must learn these techniques to estimate and model the effects of dyad-based interventions accurately.

\section{Unanswered Questions}

Throughout this paper, we have deliberately refrained from calling our framework a model. Merely identifying and organizing the relevant levels of analysis, as we have done here, does not a model make. The main contribution of the framework we have described is to highlight the role of the dyadic interaction as a mediator and moderator of the effects that several other individual-level and structural-level variables are likely to have on the enactment of safer sex behaviors within a given context. The framework suggests that the dyadic interaction must feature prominently in the models to come. However, the specific paths in future models, and the specific ways that variables at different levels of analysis fit together, still need to be formulated.

This leaves some pressing questions unanswered. One has been alluded to throughout this paper: How exactly do qualities of the relationship context affect the ability and motivation of dyads to engage in safer sex? The framework suggests that there should be powerful associations between a couple's relationship context and their dyadic capacity to coordinate safer sex. The direction of this association, however, remains in question. On one hand, we have argued that more committed, more intimate, and higher quality relationships should provide a context that strengthens the dyadic capacity to coordinate safer sex successfully. On the other hand, there is considerable empirical evidence that as intimate relationships develop, dyads often take more risks together, including having unprotected sex (e.g., contraceptive switch) [91].

Research on adolescent sexual behavior highlights this contradiction especially clearly [92]. One study, for example, found that $52 \%$ of female adolescents who had just met their sexual partner reported using no method of contraception compared with $24 \%$ of girls who reported they were "going steady" with their partner [93]. This result seems to suggest that the presence of a committed relationship facilitates safer sex. Yet another study found no difference in rates of self-reported condom use between those who had a single partner and those who were sexually active with multiple partners [94]. And still another study found that, among sexually active adolescents involved in exclusive romantic relationships, condom use declined as the length of the relationship increased [95]. Indeed, evidence from ethnographic and longitudinal research suggests that the predictors of condom use early in a relationship may differ from the predictors later in the same relationship [96]. 
How are we to reconcile these apparently contradictory sets of results? The framework described here offers no answers. However, by drawing attention to the relational context of dyadic interactions, it suggests where the answers are likely to lie. Early in a relationship, when couples are initiating sexual activity for the first time, they may be more comfortable discussing and enacting safer sex behaviors with their partners than when they feel closer and greater trust. Over time, however, deepening trust in a partner may ironically encourage couples in established relationships to be less vigilant about condom use than partners in newer relationships. Thus, recognizing how attitudes toward safer sex may evolve as the relationship between two members in a dyad develops offers a specific strategy for intervention (e.g., disentangling the motivation to engage in safer sex from concerns about the partner) along with a method of deciding when and for which couples such a strategy would be most appropriate. In the future, models of HIV-prevention will be more successful if they take the dyadic context of HIV-transmission more fully into account.

\section{Acknowledgments}

We gratefully acknowledge James Jaccard (Florida International University), David Perez-Jimenez (University of Puerto Rico), and Gail E. Wyatt (UCLA), who contributed significantly to the discussions that led to the writing of this article. This manuscript was supported by U.S. Public Health Service grant R13-MH080619 and benefited from comments provided by attendees at the funded conference.

\section{References}

1. Piot P, Bartos M, Larson H, Zewdie D, Mane P. Coming to terms with complexity: a call to action for HIV prevention. Lancet. 2008; 372(9641):845-59. [PubMed: 18687458]

2. Centers for Disease Control and Prevention. HIV/AIDS Surveillance Report: Cases of HIV Infection and AIDS in the United States and Dependent Areas, 2006. Atlanta: U.S. Department of Health and Human Services, Centers for Disease Control and Prevention; 2008.

3. Royce RA, Sena A, Cates W, Cohen MS. Sexual Transmission of HIV. N Engl J Med. 1997; 336:1072-8. [PubMed: 9091805]

4. Kelley, HH.; Berscheid, E.; Christensen, A., et al., editors. Close relationships. New York: W. H. Freeman and Company; 1983.

5. Kashy, DA.; Kenny, DA. The analysis of data from dyads and groups. In: Reis, HT.; Judd, CM., editors. Handbook of research methods in social psychology. New York: Cambridge University Press; 2000. p. 451-77.

6. Reis HT, Collins WA, Berscheid E. The relationship context of human behavior and development. Psychol Bull. 2000; 126:844-72. [PubMed: 11107879]

7. Kelley, HH.; Berscheid, E.; Christensen, A., et al. Analyzing close relationships. In: Kelley, HH.; Berscheid, E.; Christensen, A., et al., editors. Close relationships. New York: W. H. Freeman and Company; 1983. p. 20-67.

8. Albarracín D, Johnson BT, Fishbein M, Muellerleile PA. Theories of reasoned action and planned behavior as models of condom use: a meta-analysis. Psychol Bull. 2001; 127:142-61. [PubMed: 11271752]

9. Johnson BT, Carey MP, Marsh KL, Levin KD, Scott-Sheldon LAJ. Interventions to reduce sexual risk for the human immunodeficiency virus in adolescents, 1985-2000: a research synthesis. Arch Pediatr Adolesc Med. 2003; 157:381-8. [PubMed: 12695235]

10. Johnson WD, Holtgrave DR, McClellan WM, Flanders WD, Hill AN, Goodman M. HIV Intervention research for men who have sex with men: a 7-year update. AIDS Educ Prev. 2005; 17(6):568-89. [PubMed: 16398578]

11. Medley A, Kennedy C, O'Reilly K, Sweat M. Effectiveness of peer education interventions for HIV prevention in developing countries: a systematic review and meta-analysis. AIDS Educ Prev. 2009; 21(3):181-206. [PubMed: 19519235] 
12. Noar SM. Efficacy of computer technology-based HIV prevention interventions: a meta-analysis. AIDS. 2009; 23(1):107-15. [PubMed: 19050392]

13. Smoak ND, Scott-Sheldon LAJ, Johnson BT, Carey MP. SHARP Research Team. Sexual risk reduction interventions do not inadvertently increase the overall frequency of sexual behavior: a meta-analysis of 174 studies with 116,735 participants. JAIDS. 2006; 41(3):374-84. [PubMed: 16540941]

14. El-Bassel N, Witte SS, Gilbert L, et al. The efficacy of a relationship-based HIV/STD prevention program for heterosexual couples. Am J Public Health. 2003; 93:963-9. [PubMed: 12773363]

15. Sullivan PS, Salazar L, Buchbinder S, Sanchez TH. Estimating the proportion of HIV transmissions from main sex partners among men who have sex with men in five US cities. AIDS. 2009; 23(9):1153-62. [PubMed: 19417579]

16. Canin L, Dolcini MM, Adler NE. Barriers to and facilitators of HIV-STD behavior change: interpersonal and relationship-based factors. Rev Gen Psychol. 1999; 3(4):338-71.

17. Coates, DL. The cultured and culturing aspects of romantic experience in adolescence. In: Furman, W.; Brown, BB.; Feiring, C., editors. The development of romantic relationships in adolescence. New York, NY: Cambridge University Press; 1999. p. 330-63.

18. Harman JJ, Amico KR. The relationship-oriented information-motivation-behavioral skills Model: a multilevel structural equation model among dyads. AIDS Behav. 2009; 13:173-84. [PubMed: 18259851]

19. Misovich SJ, Fisher JD, Fisher WA. Close relationships and elevated HIV risk behavior: evidence and possible underlying psychological processes. Rev Gen Psychol. 1997; 1:72-107.

20. Ariely D, Lowenstein G. The heat of the moment: the effect of sexual arousal on sexual decision making. J Behav Decis Mak. 2006; 19:87-98.

21. NIMH Multisite HIV/STD Prevention Trial for African American Couples Group. Methodological overview of an African American couple-based HIV/STD prevention trial. JAIDS. 2008; 49(1):S3-13. [PubMed: 18724188]

22. NIMH Multisite HIV/STD Prevention Trial for African American Couples Group. Eban HIV/STD risk reduction intervention: conceptual basis and procedures. JAIDS. 2008; 49(1):S15-27. [PubMed: 18724186]

23. NIMH Multisite HIV/STD Prevention Trial for African American Couples Group. Formative study to develop the Eban treatment and comparison interventions for couples. JAIDS. 2008; 49(1):S4251. [PubMed: 18724190]

24. Padian NS, O'Brien TR, Chang YC, Glass S, Francis D. Prevention of heterosexual transmission of human immunodeficiency virus through couple counseling. JAIDS. 1993; 6:1043-8.

25. Parsons JT, Huszti HC, Crudder SO, Rich L, Mendoza J. Maintenance of safer sexual behaviors: evaluation of a theory-based intervention for HIV seropositive men with hemophilia and their female partners. Hemophilia. 2000; 6:181-90.

26. Harvey SM, Bird ST, Galavotti C, Duncan EAW, Greenberg D. Relationship power, sexual decision-making and condom use among women at risk for HIV/STDs. Women Health. 2002; 36:69-84. [PubMed: 12555803]

27. Harvey SM, Kraft JM, West SG, Taylor AB, Pappas-DeLuca KA, Beckman LJ. Effects of a health behavior change model-based HIV/STI prevention intervention on condom use among heterosexual couples: a randomized trial. Health Educ Behav. 2009; 36(5):878-94. [PubMed: 18784350]

28. Kraft JM, Harvey SM, Thorburn S, Hederson JT, Posner SF, Galavotti C. Intervening with couples: assessing contraceptive outcomes in a randomized pregnancy and HIV/STD risk reduction intervention trial. Women's Health Issues. 2007; 17:52-60. [PubMed: 17321948]

29. Fishbein M. The role of theory in HIV prevention. AIDS Care. 2000; 12:273-8. [PubMed: 10928203]

30. Fisher, JD.; Fisher, WA. Theoretical approaches to individual-level change in HIV behavior. In: Peterson, JL.; DiClemente, RJ., editors. Handbook of HIV prevention. New York, NY: Kluwer Academic; 2000. p. 3-56. 
31. Noar SM, Zimmerman RS. Health behavior theory and cumulative knowledge regarding health behaviors: are we moving in the right direction? Health Educ Res Theory Practice. 2005; 20(3): 275-90.

32. Bradbury, TN.; Karney, BR. Intimate relationships. New York: W. W. Norton; 2010.

33. Bronfenbrenner U. Toward an experimental ecology of human development. Am Psychol. 1977; 32:513-31.

34. Albarracín D, Gillette J, Earl A, Glasman LR, Durantini MR, Ho MH. A test of major assumptions about behavior change: a comprehensive look at the effects of passive and active HIV-prevention interventions since the beginning of the epidemic. Psychol Bull. 2005; 131:856-97. [PubMed: 16351327]

35. Simpson JA, Winterheld HA, Rholes WS, Oriäna MM. Working models of attachment and reactions to different forms of caregiving from romantic partners. J Pers Soc Psychol. 2007; 93(3): 466-77. [PubMed: 17723060]

36. Amaro H. Love, sex, and power: Considering women's realities in HIV prevention. Am Psychol. 1995; 50:437-43. [PubMed: 7598292]

37. Wingood GM, DiClemente RJ. Understanding the role of gender relations in HIV prevention research. Am J Public Health. 1995; 85:592. [PubMed: 7702134]

38. Simpson, JA. Foundations of interpersonal trust. In: Kruglanski, AW.; Higgins, ET., editors. Social psychology: handbook of basic principles. 2. New York: Guilford; 2007. p. 587-607.

39. Reis, HT.; Shaver, P. Intimacy as an interpersonal process. In: Duck, SW., editor. Handbook of personal relationships: theory, research and interventions. Chichester, England: Wiley; 1988. p. 367-89.

40. Ickes, W.; Simpson, JA. Motivational aspects of empathic accuracy. In: Fletcher, GJO.; Clark, M., editors. The Blackwell handbook in social psychology: interpersonal processes. Oxford, England: Blackwell; 2001. p. 229-49.

41. Berscheid, E.; Reis, HT. Attraction and close relationships. In: Gilbert, DT.; Fiske, ST.; Lindzey, G., editors. The handbook of social psychology. New York, NY: McGraw-Hill; 1998. p. 193-281.

42. Rusbult CE, Buunk BP. Commitment processes in close relationships: an interdependence analysis. J Soc Pers Relatsh. 1993; 10:175-204.

43. Van Lange PAM, Rusbult CE, Drigotas SM, Arriaga XB, Witcher BS, Cox CL. Willingness to sacrifice in close relationships. J Pers Soc Psychol. 1997; 72(6):1373-95. [PubMed: 9177022]

44. Huston, TL. Power. In: Kelley, HH.; Berscheid, E.; Christensen, A., et al., editors. Close relationships. San Francisco: Freeman; 1983. p. 169-219.

45. Karney BR, Bradbury TN. The longitudinal course of marital quality and stability: a review of theory, methods, and research. Psychol Bull. 1995; 118(1):3-34. [PubMed: 7644604]

46. Remien RH, Wagner GJ, Dolezal C, Carballo-Dieguez A. Factors associated with HIV sexual risk behavior in male couples of mixed HIV status. J Psychol Human Sex. 2001; 13(2):31-47.

47. Wagner GJ, Remien RH, Carballo-Dieguez A. Prevalence of extradyadic sex in male couples of mixed HIV status and its relationship to psychological distress and relationship quality. $\mathrm{J}$ Homosex. 2000; 39:31-46. [PubMed: 10933280]

48. Doherty IA, Schoenbach VJ, Adimora AA. Sexual mixing patterns and heterosexual HIV transmission among African Americans in the southeastern United States. JAIDS. 2009; 52(1): 114-20. [PubMed: 19506485]

49. Magnus M, Kuo I, Shelley K, et al. Risk factors driving the emergence of a generalized heterosexual HIV epidemic in Washington, District of Columbia networks at risk. AIDS. 2009; 23(10):1277-84. [PubMed: 19440142]

50. Lenior CD, Adler NE, Borzekowski G, Tschann JM, Ellen JM. What you don't know can hurt you: perceptions of sex-partner concurrency and partner-reported behavior. J Adolesc Health. 2006; 38:179-85. [PubMed: 16488813]

51. Epstein, H. The invisible cure: Africa, the west, and the fight against AIDS. New York: Farrar Straus \& Giroux; 2007.

52. Leclerc-Madlala S. Cultural scripts for multiple and concurrent partnerships in southern Africa: Why HIV prevention needs anthropology. Sex Health. 2009; 6(2):103-10. [PubMed: 19457288] 
53. Fishbein, M.; Ajzen, I. Belief, Attitude, Intention, and Behavior: an Introduction to Theory and Research. Reading, MA: Addison-Wesley; 1975.

54. Kenny, DA.; Kashy, DA.; Cook, WL. Dyadic data analysis. New York: Guilford; 2006.

55. Ajzen, I.; Fishbein, M. Understanding attitudes and predicting social behavior. Englewood Cliffs, NJ: Prentice-Hall; 1980.

56. Rusbult, CE.; van Lange, PAM. Interdependence processes. In: Higgins, ET.; Kruglanski, A., editors. Social psychology: handbook of basic principles. New York: Guilford; 1996. p. 564-96.

57. CAPS. Center for AIDS Prevention Studies. University of California; San Francisco: 2003. HIV prevention fact sheets: Family.

58. Aquilino WS. From adolescent to young adult: a prospective study of parent-child relations during the transition to adulthood. J Marriage Fam. 1997; 59:670-86.

59. Bucx F, van Wel F, Knijn T, Hagendoorn L. Intergenerational contact and the life course status of young adult children. J Marriage Fam. 2008; 70(1):144-56.

60. MacMillan R, Copher R. Families in the life course: interdependency of roles, role configurations, and pathways. J Marriage Fam. 2005; 67:858-79.

61. Tee K, Bernheim Brush AJ, Inkpen KM. Exploring communication and sharing between extended families. Int J Human-Comput Stud. 2009; 67:128-38.

62. Parke RD. Development in the family. Annu Rev Psychol. 2004; 55:365-9. [PubMed: 14744220]

63. Fasula AM, Miller KS. African-American and Hispanic adolescents' intentions to delay first intercourse: parental communication as a buffer for sexually active peers. J Adolesc Health. 2006; 38:193-200. [PubMed: 16488815]

64. Trejos-Castillo E, Vazsonyi AT. Risky sexual behaviors in first and second generation Hispanic immigrant youth. J Youth Adolesc. 2009; 38:719-31. [PubMed: 19636766]

65. Perrino T, Gonzalez-Soldevilla A, Pantin H, Szapocznik J. The role of families in adolescent HIV prevention: a review. Clin Child Fam Psychol Rev. 2000; 3(2):81-96. [PubMed: 11227063]

66. Kirby, D.; Lepore, G.; Ryan, J. Factors affecting teen sexual behavior, pregnancy, childbearing and sexually transmitted disease: Which are important? Which can you change?. Washington, D. C: The National Campaign to Prevent Teen Pregnancy; 2005. Sexual risk and protective factors.

67. Andrews JA, Tildesley E, Hops H, Li F. The influence of peers on young adult substance use. Health Psychol. 2002; 21:349-57. [PubMed: 12090677]

68. Hops, H.; Andrews, JA.; Duncan, SC.; Duncan, TE.; Tildesley, E. Adolescent drug use development: a social interactional and contextual perspective. In: Sameroff, AJ.; Lewis, M.; Miller, SM., editors. Handbook of developmental psychopathology. 2. New York: Kluwer Academic/Plenum; 2000. p. 589-605.

69. Leonard KE, Kearns J, Mudar P. Peer networks among heavy, regular and infrequent drinkers prior to marriage. J Stud Alcohol. 2000; 61:669-73. [PubMed: 11022805]

70. Leonard KE, Mudar P. Peer and partner drinking and the transition to marriage: a longitudinal examination of selection and influence processes. Psychol Addict Behav. 2003; 17:115-25. [PubMed: 12814275]

71. Karney, BR.; Beckett, MK.; Collins, RL.; Shaw, RN. Adolescent romantic relationships as precursors of healthy adult marriages: a review of theory, research, and programs. Santa Monica, CA: RAND Corporation; 2007. Report No.: TR433-ACF

72. Connolly J, Furman W, Konarski R. The role of peers in the emergence of heterosexual romantic relationships in adolescence. Child Develop. 2000; 71(5):1395-408. [PubMed: 11108103]

73. Furman W, Simon VA, Shaffer L, Bouchey HA. Adolescents' working models and styles for relationships with parents, friends, and romantic partners. Child Develop. 2002; 73:241-55. [PubMed: 14717255]

74. Collins, WA.; Hennighausen, KC.; Schmit, DT.; Sroufe, LA. Developmental precursors of romantic relationships: a longitudinal analysis. In: Shulman, S.; Collins, WA., editors. Romantic relationships in adolescence: developmental perspectives. San Francisco, CA: Jossey-Bass; 1997. p. 69-84.

75. Seiffge-Krenke IJ, Shulman S, Klessinger N. Adolescent precursors of romantic relationships in young adulthood. J Soc Pers Relatsh. 2001; 18(3):327-46. 
76. Kinsfogel KM, Grych JH. Interparental conflict and adolescent dating relationships: integrating cognitive, emotional, and peer influences. J Fam Psychol. 2004; 18:505-15. [PubMed: 15382975]

77. Centers for Disease Control and Prevention. Sexual and reproductive health of persons aged 10-24 years-United States, 2002-2007. Morbidity and Mortality Weekly Report 2009. 2009; 58(SS-6)

78. Centers for Disease Control and Prevention. Surveillance Summaries MMWR. 2004; 53(SS-2)

79. Moreno CL, El-Bassell N, Morrill AC. Heterosexual women of color and HIV risk: sexual risk factors for HIV among Latina and African-American women. Women Health. 2009; 45:1-15. [PubMed: 18032159]

80. Centers for Disease Control and Prevention. Youth Risk Behavior Surveillance-United States, 2001. Morbidity and Mortality Weekly Report. 2002; 51:SS-4.

81. Grunbaum JA, Kann L, Kinchen S, et al. Youth risk behavior surveillance-United States, 2003 (Abridged). J School Health. 2004; 74:307-24. [PubMed: 15554117]

82. Kann L, Kinchen SA, Williams BI, et al. Special report: youth risk behavior surveillance-United States, 1999. J School Health. 2000; 70:271-85. [PubMed: 10981282]

83. Pantin H, Prado G, Lopez B, et al. A randomized controlled trial of Familias Unidas for Hispanic adolescents with behavior problems. Psychosom Med. 2009; 71(9):987-95. [PubMed: 19834053]

84. Villarruel AM, Jemmott JB, Jemmott LS. A randomized controlled trial testing and HIV prevention intervention for Latino youth. Arch Pediatr Adolesc Med. 2006; 160:772-7. [PubMed: 16894074]

85. Peragallo N. Latina women and AIDS risk. Public Health Nurs. 1996; 13(3):217-22. [PubMed: 8677238]

86. Hops, H.; Ozechowski, T.; Waldron, HB.; Davis, BT.; Turner, CT.; Brody, J. Adolescent healthrisk sexual behaviors: effects of a drug abuse intervention. 2010. submitted

87. Jemmott JB, Jemmott LS, Fong GT, McCaffree K. Reducing HIV risk-associated sexual behavior among African American adolescents: testing the generality of intervention effects. Am J Community Psychol. 1999; 27:161-87. [PubMed: 10425698]

88. Whaley AL, Davis KE. Cultural competence and evidence-based practice in mental health services. Am Psychol. 2007; 62(6):563-74. [PubMed: 17874897]

89. Jacobson NS, Moore D. Spouses as observers of the events in their relationship. J Consult Clin Psychol. 1981; 49:269-77. [PubMed: 7217493]

90. Witte SS, El-Bassel N, Gilbert L, Wu E, Chang M. Predictors of discordant reports of sexual and $\mathrm{HIV} /$ sexually transmitted infection risk behaviors among heterosexual couples. Sex Transm Dis. 2007; 34(5):302-8. [PubMed: 17016237]

91. Noar, SM.; Zimmerman, RS.; Atwood, KA. Safer sex and sexually transmitted infections from a relationship perspective. In: Harvey, J.; Wenzel, A.; Sprecher, S., editors. Handbook of sexuality in close relationships. Mahwah, NJ: Lawrence Erlbaum; 2004.

92. Giordano PC. Relationships in adolescence. Annu Rev Sociol. 2003; 29:257-81.

93. Manning W, Longmore M, Giordano P. The relationship context of contraceptive use at first intercourse. Fam Plann Perspect. 2000; 32(3):104-10. [PubMed: 10894255]

94. Lescano CM, Vazquez EA, Brown LK, Litvin EB, Pugatch D. Condom Use with "Casual" and "Main" Partners: What's in a Name? J Adolesc Health. 2006; 39(3):e1-7.

95. Ku L, Sonenstein FL, Pleck JH. The dynamics of young men's condom use during and across relationships. Fam Plann Perspect. 1994; 26:246-51. [PubMed: 7867771]

96. Sayegh MA, Fortenberry JD, Shew M, Orr DP. The developmental association of relationship quality, hormonal contraceptive choice and condom non-use among adolescent women. J Adolesc Health. 2006; 39(3):388-95. [PubMed: 16919801] 


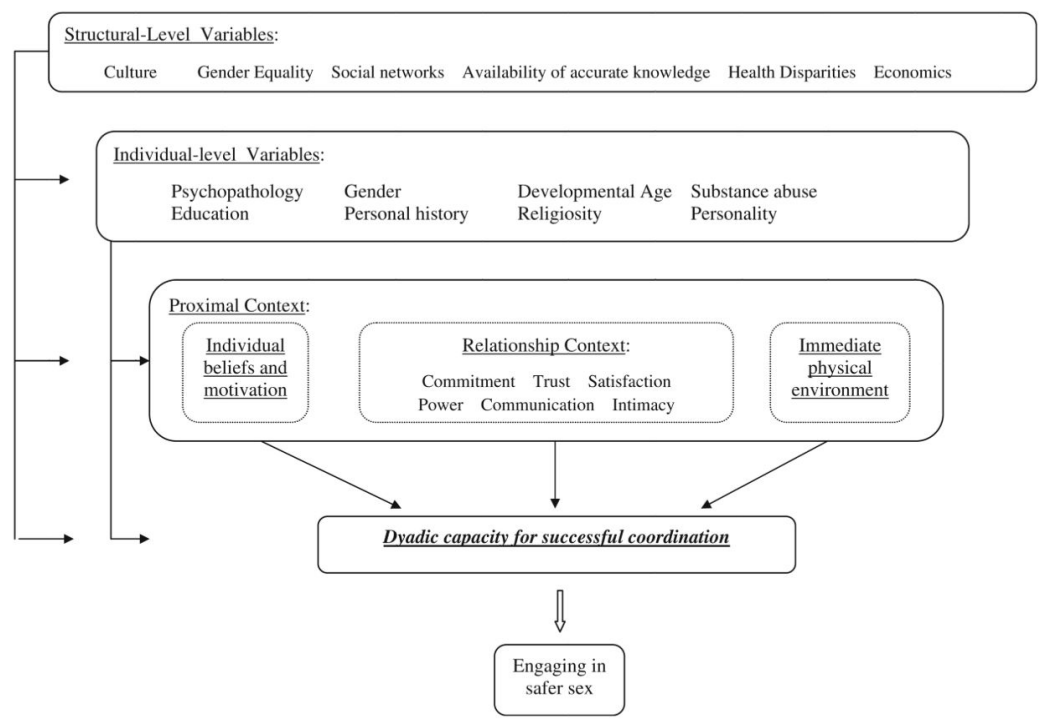

Fig. 1.

A Dyadic Framework for Incorporating Dyads in HIV Prevention. Note: The specific structural, individual, and relational variables within each box of the figure are provided as examples of each type of variable and should not be read as an exhaustive list 
Person A:

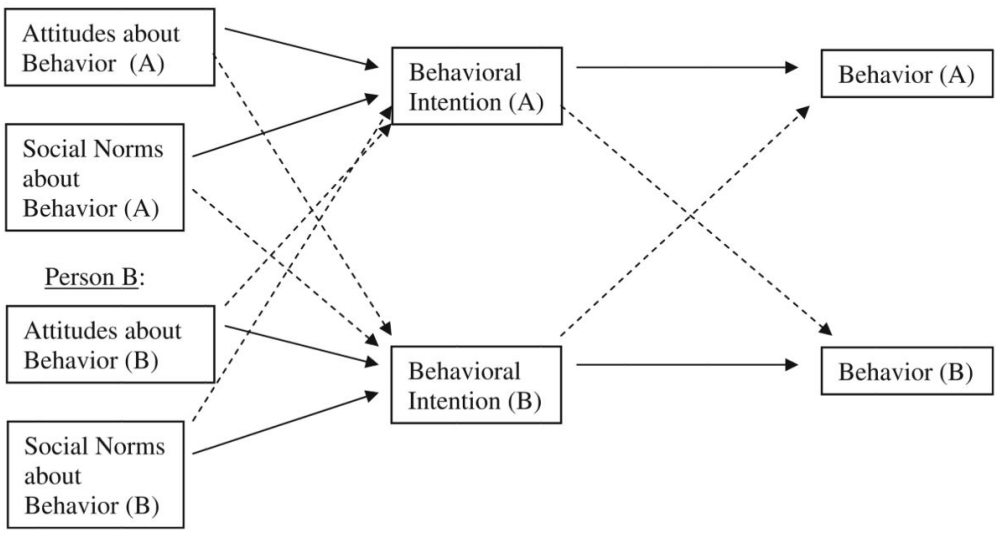

Fig. 2.

Dyadic Model for two persons (Person A and Person B). Note: Solid lines are actor effects derived from an individual-based model (e.g., TRA); dashed lines are partner effects derived from a dyadic-based interpretation of the model. This version of the model describes behaviors that each member of the dyad can enact separately (e.g., HIV testing) 


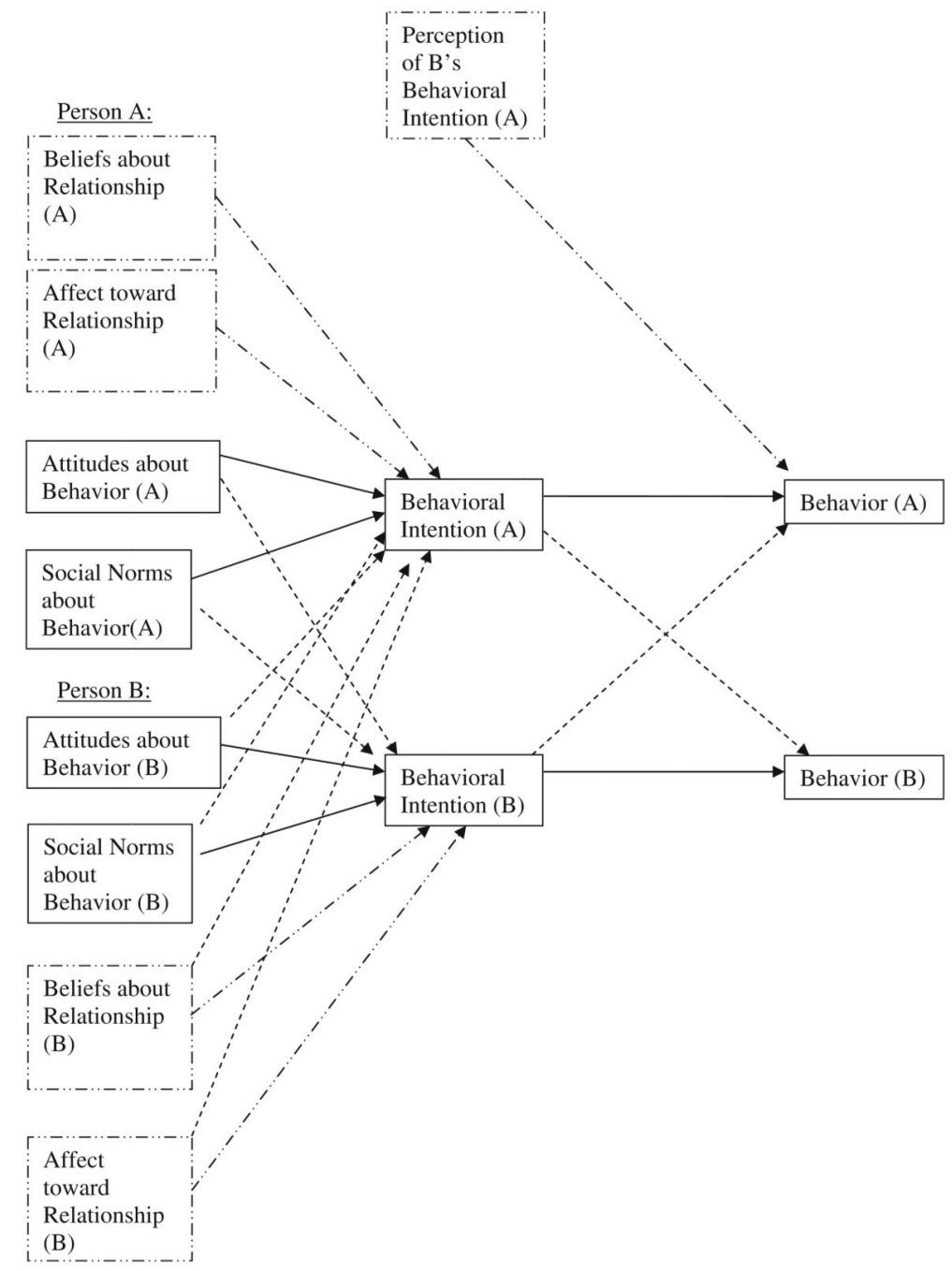

Fig. 3.

Incorporating relationship-specific constructs within a dyadic model. Note: Solid lines represent actor effects and dashed lines represent partner effects 